\title{
Local Lorentz Transformation and Mass-Energy Relation of Spinor
}

\author{
Ying-Qiu $\mathrm{Gu}^{*}$ \\ School of Mathematical Science, Fudan University, Shanghai 200433, China
}

(Dated: 30th May 2017)

\begin{abstract}
In this paper, we strictly establish classical concepts and relations according to a Dirac equation with scalar, vector and nonlinear potentials. To calculate classical parameters for moving spinor, the local Lorentz transformations for parameters are derived. The calculation shows that different kinds of potentials result in different energy-speed relations, and the energy-speed relations for these potentials are derived in detail. The usual mass-energy relation $E=m c^{2}$ holds only for the linear spinor. The energy-speed relations can be used as fingerprints to identify the interactive potentials of a particle by elaborated experiments. The analysis and results of this paper can also provide some natural explanations for the foundation of quantum mechanics, and clarify some long-standing puzzles in the theory.
\end{abstract}

Keywords: mass-energy relation, classical approximation, local Lorentz transformation, foundation of quantum mechanics

PACS numbers: $03.65 . T a$, 03.50.Kk, 03.65.Pm, 11.10.Lm

\section{INTRODUCTION}

Einstein's mass-energy relation

$$
E=m c^{2}, \quad m=m_{0}\left(1-\frac{v^{2}}{c^{2}}\right)^{-\frac{1}{2}},
$$

is the apotheosis of the elegance and simplicity of modern science, which becomes a cornerstone of the modern physics. However its original derivation is based on an idealized model and valid only for linear theories[1]. For a spinor with self-potential, the energy-speed relation includes fine structure[2], namely, the energy caused by potentials differs from (1.1).

*Electronic address: yqgu@fudan.edu.cn 
In $E=m c^{2}$, the total mass $m$ should weakly depend on the speed $v$ of the particle, and a different kind of potential leads to a different energy-speed relation. So it is meaningful to derive the detailed energy-speed relation for each kind potential. These relations can be used as the fingerprints of the interactive potentials, and can be measured by elaborated experiments.

This problem is also closely related with some important puzzles in fundamental physics as listed in [3], that is, the consistence of general relativity and quantum theory, the foundation of quantum mechanics, the unification for the particles and interactions, dark matter and dark energy, and so on. Among these puzzles the foundation of quantum mechanics is the key. From its beginning, quantum mechanics provides us counterintuitive concepts and pictures for the world such as the principle of the uncertainty, wave function of probability, correspondence principle of operators with parameters. These concepts have greatly changed our world outlook. However, with its continuous and tremendous successes, quantum mechanics also makes troubles continually for us.

To explain the foundation of quantum mechanics, we should clarify the relationship between quantum mechanics with classical mechanics at first. In the textbooks we have a standard procedure. By correspondence principle, a classical parameter such as energy or momentum corresponds to an operator, and the operator acts on state functions to form a dynamics in quantum mechanics. On the contrary, by calculating the mean value of an operator we get the classical approximation for the parameter. Such parallel relation easily results in confusion in logic.

In this paper, we take a generalized Dirac equation with nonlinear, scalar and vector interactive potentials as example to show the local Lorentz transformation for classical parameters and derive complete classical mechanics and energy-speed relations for the spinor. These derivations and results imply a natural explanation for the relationship between classical mechanics and quantum mechanics. We find some puzzles may be avoidable. At last, a crucial experiment is proposed. 


\section{LOCAL LORENTZ TRANSFORMATION LAWS FOR CLASSICAL PARAMETERS}

First, we introduce some notations and conventions. Taking the Minkowski metric as $\eta_{\mu \nu}=\operatorname{diag}(1,-1,-1,-1)$, and $c=1$ as the unit of speed. Pauli matrices are given by

$$
\vec{\sigma}=\left(\sigma^{k}\right)=\left\{\left(\begin{array}{ll}
0 & 1 \\
1 & 0
\end{array}\right),\left(\begin{array}{cc}
0 & -i \\
i & 0
\end{array}\right),\left(\begin{array}{cc}
1 & 0 \\
0 & -1
\end{array}\right)\right\} .
$$

Instead of Dirac matrices $\gamma^{\mu}$, define $4 \times 4$ Hermitian matrices as follows for the convenience of calculation,

$$
\alpha^{\mu}=\left\{\left(\begin{array}{cc}
I & 0 \\
0 & I
\end{array}\right),\left(\begin{array}{cc}
0 & \vec{\sigma} \\
\vec{\sigma} & 0
\end{array}\right)\right\}, \quad \gamma=\left(\begin{array}{cc}
I & 0 \\
0 & -I
\end{array}\right) .
$$

We use Greek characters stand for 4-vector index and Latin characters for spatial index.

Considering the following nonlinear spinor equation

$$
\alpha^{\mu}\left(\hbar i \partial_{\mu}-e A_{\mu}\right) \phi=\left(\mu c-F^{\prime}\right) \gamma \phi
$$

where the coefficient $\mu>0$ is a constant mass, $F(\check{\gamma})>0$ is the nonlinear function of the quadratic scalar $\check{\gamma} \equiv \phi^{+} \gamma \phi$. Solutions to (2.3) have particle-wave duality. Some numeric simulations were provided in [4]-[9].

For Dirac equation (2.3), we have current conservation law due to the gauge invariance

$$
\partial_{\mu} \rho^{\mu}=0, \quad \rho^{\mu}=\phi^{+} \alpha^{\mu} \phi,
$$

which leads to the normalizing condition

$$
\int_{R^{3}} \rho^{0} d^{3} x=1
$$

Due to (2.4) and (2.5), we can define classical concepts for the spinor $\phi$.

Definition 1. For spinor field $\phi$, we define the coordinate $\vec{X}$ and velocity $\vec{v}$ respectively by

$$
\vec{X}(t)=\int_{R^{3}} \vec{x} \rho^{0} d^{3} x, \quad \vec{v}=\frac{d}{d t} \vec{X},
$$

where $t=x^{0}$. The coordinate system with origin $\vec{X}=0$ is called the central coordinate system of the spinor.

Lemma 1. For a spinor, we have

$$
\vec{v}=\int_{R^{3}} \vec{\rho} d^{3} x
$$


Proof. By the current conservation law (2.4), we have

$$
\vec{v}=\int_{R^{3}} \vec{x} \partial_{0} \rho^{0} d^{3} x=-\int_{R^{3}} \vec{x} \nabla \cdot \vec{\rho} d^{3} x=\int_{R^{3}} \vec{\rho} d^{3} x .
$$

By (2.5) and (2.7), we have the classical approximation of the spinor, i.e.

Definition 2. if the moving scale of a spinor is much larger than its mean radius $\bar{r}=\int|\vec{x}-\vec{X}| \rho^{0} d^{3} x$, we have point-particle model for the spinor,

$$
\rho^{\mu} \rightarrow u^{\mu} \sqrt{1-v^{2}} \delta(\vec{x}-\vec{X})
$$

where $u^{\mu}$ is the 4 -vector speed

$$
u^{\mu} \equiv(\xi, \xi \vec{v}), \quad \xi=\frac{1}{\sqrt{1-v^{2}}} .
$$

(2.9) is the origin of the classical models 'mass-point' and 'point-charge'[2, 10]. The classical variables such as momentum and energy are also defined as the integrals of some Nöther charges similar to (2.8). However, in usual cases, the values depend on the spinor at energy eigen state or not, due to absence of conservation law similar to (2.4) for the spinor as a closed system. So it is necessary to distinguish the different states of the spinor.

Definition 3. If a spinor takes energy eigenstate in the central coordinate system and moves smoothly without emitting and absorbing energy quantum, we call it is at the particle state. Otherwise, the spinor is in the process of exchanging energy with its environment, we call it in the quantum process.

For a spinor at particle state moving smoothly, we can clearly define the classical parameters such as "momentum", "energy" and "mass", and then derive the classical mechanics from (2.3). But for the spinor in the quantum process, the detailed description of the process should be the original equation (2.3) and quantum theory.

In the usual case, we can calculate only the classical parameters in central coordinate system, i.e. the proper parameters. In a general coordinate system, we should derive the moving parameters by local Lorentz transformation. Since the rotational transformation is trivial, we consider only the boosting one. Considering the central coordinate system of the spinor with coordinate $\bar{x}^{\mu}$, which moves along $x^{1}$ at speed $v$, and $\bar{x}^{k}(k \neq 0)$ is parallel to $x^{k}$, so $\bar{x}^{k}=0$ corresponds to the mass center $\vec{X}^{k}(t)$ of the field $\phi$. Then the Lorentz transformation between $x^{\mu}$ and $\bar{x}^{\mu}$ in the form of matrix is given by

$$
x=L(v) \bar{x}, \quad \bar{x}=L(v)^{-1} x=L(-v) x
$$


where $x=\left(t, x^{1}, x^{2}, x^{3}\right)^{T}, \bar{x}=\left(\bar{x}^{0}, \bar{x}^{1}, \bar{x}^{2}, \bar{x}^{3}\right)^{T}$ and

$$
L(v)=\operatorname{diag}\left(\left(\begin{array}{cc}
\xi & \xi v \\
\xi v & \xi
\end{array}\right), 1,1\right)=\left(L^{\mu}{ }_{\nu}\right) .
$$

Assuming $S, P^{\mu}$ and $T^{\mu \nu}$ are any scalar, vector and tensor defined by some real functions of $\phi$ and their derivatives such as $S=\check{\gamma}^{2}, T^{\mu \nu}=\Re\left\langle\phi^{+} \alpha^{\mu} i \partial^{\nu} \phi\right\rangle$ etc., where $\Re\langle\rangle$ stands for taking real part. For a spinor at particle state, all these functions in central coordinate system are independent of proper time $\bar{x}^{0}$. So the spatial integrals of these functions define the proper classical parameters of the spinor, which are all constants. Their Lorentz transformation laws are given by

Theorem 2. For a spinor at particle state, the integrals of covariant functions $S, P^{\mu}$ and $T^{\mu \nu}$ satisfy the following instantaneous Lorentz transformation laws under the boosting transformation (2.11) between $x^{\mu}$ and $\bar{x}^{\mu}$ at $t \equiv t_{0}$ or $d t=0$,

$$
\begin{aligned}
I & \equiv \int_{R^{3}} S(x) d^{3} x=\sqrt{1-v^{2}} \bar{I} \\
I^{\mu} & \equiv \int_{R^{3}} P^{\mu}(x) d^{3} x=\sqrt{1-v^{2}} L^{\mu}{ }_{\nu} \bar{I}^{\nu}, \\
I^{\mu \nu} & \equiv \int_{R^{3}} T^{\mu \nu}(x) d^{3} x=\sqrt{1-v^{2}} L_{\alpha}^{\mu} L_{\beta}^{\nu} \bar{I}^{\alpha \beta},
\end{aligned}
$$

where $\bar{I}, \bar{I}^{\mu}, \bar{I}^{\mu \nu}$ are the proper parameters defined in the central coordinate system

$$
\bar{I}=\int_{R^{3}} S(\bar{x}) d^{3} \bar{x}, \quad \bar{I}^{\mu}=\int_{R^{3}} \bar{P}^{\mu} d^{3} \bar{x}, \quad \bar{I}^{\mu \nu}=\int_{R^{3}} \bar{T}^{\mu \nu} d^{3} \bar{x} .
$$

Proof. We take (2.14) as an example to show the proof. For a spinor at the particle state, by the transformation law of contravariant vector, we have

$$
P^{\mu}(x)=L^{\mu}{ }_{\nu} \bar{P}^{\nu}(\bar{x})=L^{\mu}{ }_{\nu} \bar{P}^{\nu}\left(\bar{x}^{1}, \bar{x}^{2}, \bar{x}^{3}\right)=P^{\mu}\left(\xi\left(x^{1}-v t\right), x^{2}, x^{3}\right) .
$$

So the integral can be calculated as follows

$$
\begin{aligned}
I^{\mu} & =\left.\int_{R^{3}} P^{\mu}(x) d^{3} x\right|_{d t=0} \\
& =\int_{R^{3}} P^{\mu}\left(\xi\left(x^{1}-v t\right), x^{2}, x^{3}\right) \sqrt{1-v^{2}} d\left[\xi\left(x^{1}-v t\right)\right] d x^{2} d x^{3} \\
& =\int_{R^{3}} L^{\mu}{ }_{\nu} \bar{P}^{\nu}(\bar{x}) \sqrt{1-v^{2}} d^{3} \bar{x}=\sqrt{1-v^{2}} L^{\mu} \bar{I}^{\nu} .
\end{aligned}
$$

The proof is finished.

For the above calculations, some explanations are given in the following: 
Remarks 1. The integration-domain of Eqs.(2.13)-(2.16) is the realistic space of the world, which is a simultaneous hypersurface $t \equiv t_{0}$ in one special coordinate system. In Ref.[11] we analyzed the existence and uniqueness of this simultaneous hypersurface in the real world. In the case of Minkowski space-time, the integration-domain becomes $R^{3}$ in any Descartes coordinate system. However, a relativistic factor $\sqrt{1-v^{2}}$ will appear in the integrals under Lorentz transformation as derived in (2.18), because the space is tilted and its measure is changed in the new coordinate system.

Remarks 2. The Lorentz transformation laws (2.13), (2.14) and (2.15) are valid for varying speed $v(t)$, because the integrals are related only to the simultaneous condition $d t=0$, and the relations are related only to algebraic calculations. Since spinor has only a tiny micro structure in contrast with the space-time, the relations Eqs.(2.13)-(2.15) actually hold in the tangent space-time $\gamma_{\alpha} \delta X^{\alpha}$ of curved manifold with the spinor central coordinate $\vec{X}=0$. So we call them local Lorentz transformation. Besides, when we consider manybody problem, each spinor has a different velocity and then involves a different Lorentz transformation.

Remarks 3. When the spinor is not at the particle state, the covariant integrands will depend on the proper time $\bar{x}^{0}$, so the calculation (2.18) becomes approximate in general cases, and consequently the relations (2.13)-(2.15) usually hold only approximately unless the integrand satisfies the conservation law similar to (2.4).

\section{THE CLASSICAL MECHANICS FOR A SPINOR AND MASS-ENERGY RELATION}

In this section, we take the following more general Lagrangian as an example to derive classical mechanics for a spinor,

$$
\begin{aligned}
\mathcal{L}= & \phi^{+} \alpha^{\mu}\left(\hbar i \partial_{\mu}-e A_{\mu}\right) \phi-\mu \check{\gamma}+F(\check{\gamma})-s \check{\gamma} G \\
& -\frac{1}{2} \partial_{\mu} A_{\nu} \partial^{\mu} A^{\nu}-\frac{1}{2}\left(\partial_{\mu} G \partial^{\mu} G-b^{2} G^{2}\right),
\end{aligned}
$$

where $A^{\mu}$ and $G$ are the self-potential of the spinor, $F(\check{\gamma})>0$ is a concave function satisfying

$$
F^{\prime}(\check{\gamma}) \check{\gamma}>F(\check{\gamma}), \quad(\text { for } \check{\gamma}>0)
$$

The corresponding dynamical equation is given by

$$
\begin{aligned}
\alpha^{\mu}\left(\hbar i \partial_{\mu}-e A_{\mu}\right) \phi & =\left(\mu+s G-F^{\prime}\right) \gamma \phi \\
\partial_{\alpha} \partial^{\alpha} A^{\mu} & =e \rho^{\mu} \\
\left(\partial_{\alpha} \partial^{\alpha}+b^{2}\right) G & =s \check{\gamma} .
\end{aligned}
$$


The Hamiltonian form of (3.3) reads

$$
\hbar i \partial_{t} \phi=\hat{H} \phi, \quad \hat{H}=e A_{0}+\vec{\alpha} \cdot(-\hbar i \nabla-e \vec{A})+\left(\mu+s G-F^{\prime}\right) \gamma
$$

The complete dynamical equation of $A^{\mu}$ in 3 -d form is given by the following Maxwell equation $[2,12]$

$$
\begin{cases}\vec{E}=-\nabla A^{0}-\partial_{0} \vec{A}, & \vec{B}=\nabla \times \vec{A}, \\ \nabla \cdot \vec{E}=e \rho^{0}, & \nabla \times \vec{E}=-\partial_{0} \vec{B}, \\ \nabla \cdot \vec{B}=0, & \nabla \times \vec{B}=\partial_{0} \vec{E}+e \vec{\rho},\end{cases}
$$

where $\vec{A}=\left(A^{1}, A^{2}, A^{3}\right)$ is the spatial part of a contravariant vector $A^{\mu}$. For the scalar $G$ we have not similar decomposition with manifest physical meanings.

Assuming the spinor is at particle state, we can define the classical momentum and energy for the spinor according to Nöther's theorem[2, 13],

Definition 4. Define the 4-vector momentum $p^{\mu}$ and energy $E$ of the particle described by (3.1) respectively by

$$
\begin{aligned}
p^{\mu} & \equiv \int_{R^{3}} \phi_{k}^{+}\left(\hbar i \partial^{\mu}-e A^{\mu}\right) \phi d^{3} x \\
E & \equiv \int_{R^{3}}\left(\sum_{\forall f} \frac{\partial \mathcal{L}}{\partial\left(\partial_{t} f\right)} \partial_{t} f-\mathcal{L}\right) d^{3} x=p^{0}+E_{F}+E_{A}+E_{G}
\end{aligned}
$$

in which

$$
\begin{aligned}
E_{F} & =\int_{R^{3}}\left(F^{\prime} \check{\gamma}-F\right) d^{3} x, \\
E_{A} & =-\frac{1}{2} \int_{R^{3}}\left(\partial_{0} A_{\mu} \partial^{0} A^{\mu}+\nabla A_{\mu} \cdot \nabla A^{\mu}-2 e \rho_{0} A^{0}\right) d^{3} x, \\
E_{G} & =-\frac{1}{2} \int_{R^{3}}\left(\partial_{0} G \partial^{0} G+\nabla G \cdot \nabla G+b^{2} G^{2}\right) d^{3} x,
\end{aligned}
$$

where we take the spinor as a closed system, and $A^{\mu}$ and $G$ are self-potentials satisfying the natural boundary condition.

In the central coordinate system $\bar{x}^{\mu}$ of the spinor, since the spinor takes the energy eigenstate, we have $\partial_{0} A^{\mu}=\partial_{0} G=0$. By the Green's functions of (3.4) and (3.5), we can 
calculate the corresponding static energy as follows

$$
\begin{aligned}
W_{F} & =\int_{R^{3}}\left(F^{\prime} \check{\gamma}-F\right) d^{3} \bar{x}>0, \\
W_{A} & =\frac{1}{2} \int_{R^{3}}\left(A_{\mu} \Delta A^{\mu}+2 e \rho_{0} A^{0}\right) d^{3} \bar{x} \\
& =\frac{1}{2} e \int_{R^{3}}\left(\rho_{0} A^{0}+\vec{\rho} \cdot \vec{A}\right) d^{3} \bar{x} \\
& =\frac{e^{2}}{8 \pi} \int_{R^{6}} \frac{1}{r}\left[|\phi(\bar{x})|^{2}|\phi(\bar{y})|^{2}+\vec{\rho}(\bar{x}) \cdot \vec{\rho}(\bar{y})\right] d^{3} \bar{x} d^{3} \bar{y} \\
& \doteq \frac{e^{2}}{8 \pi} \int_{R^{6}} \frac{1}{r}|\phi(\bar{x})|^{2}|\phi(\bar{y})|^{2} d^{3} \bar{x} d^{3} \bar{y}, \\
W_{G} & =\frac{1}{2} \int_{R^{3}} G\left(\Delta-b^{2}\right) G d^{3} \bar{x}=-\frac{1}{2} s \int_{R^{3}} \check{\gamma} G d^{3} \bar{x} \\
& =-\frac{s^{2}}{8 \pi} \int_{R^{6}} \frac{\exp (-b r)}{r} \check{\gamma}(\bar{x}) \check{\gamma}(\bar{y}) d^{3} \bar{x} d^{3} \bar{y},
\end{aligned}
$$

where $r=|\bar{x}-\bar{y}|$. By (3.14) and (3.15), we find that $W_{A}$ provides positive self-energy, but $W_{G}$ provides negative one, so the scalar field is quite different from the vector one in some aspects.

According to theorem 2, making local Lorentz transformation, we get energy-speed relation for each part in (3.9) for a moving spinor $\phi$ as follows,

$$
\begin{aligned}
& E_{F}=W_{F} \sqrt{1-v^{2}} \\
& E_{A} \doteq W_{A}\left(\frac{2}{\sqrt{1-v^{2}}}-\sqrt{1-v^{2}}-\frac{2 v^{2}}{3 \sqrt{1-v^{2}}}\right) \\
& E_{G}=W_{G}\left(\sqrt{1-v^{2}}+\frac{2 v^{2}}{3 \sqrt{1-v^{2}}}\right)-W_{b} \frac{v^{2}}{\sqrt{1-v^{2}}} .
\end{aligned}
$$

where

$$
W_{b}=\frac{1}{3}\left(\frac{b s}{4 \pi}\right)^{2} \int_{R^{3}}\left(\int_{R^{3}} \frac{\exp (-b r)}{r} \check{\gamma}(\bar{y}) d^{3} \bar{y}\right)^{2} d^{3} \bar{x} .
$$

Now we examine the term $p^{\mu}$. It is easy to check the following Ehrenfest theorem.

Lemma 3. For any Hermitian operator $\hat{P}$ and corresponding classical quantity $P$ for the spinor defined by

$$
P \equiv \int_{R^{3}} \phi^{+} \hat{P} \phi d^{3} x
$$

we have

$$
\frac{d}{d t} P=\int_{R^{3}} \phi^{+}\left(\partial_{t} \hat{P}+i[\hat{H}, \hat{P}]\right) \phi d^{3} x
$$


where $[\hat{H}, \hat{P}]=\hat{H} \hat{P}-\hat{P} \hat{H}$.

By Lemma 3 and the dynamical equation (3.6), we have[2]

Theorem 4. For 4-vector momentum $p^{\mu}$ defined by (3.8), we have the following rigorous dynamical equations

$$
\left\{\begin{array}{l}
\frac{d}{d t} p^{0}=\int\left(e \vec{\rho} \cdot \vec{E}+s \check{\gamma} \partial_{0} G\right) d^{3} x-\frac{d}{d t} E_{F}, \\
\frac{d}{d t} \vec{p}=\int\left[e\left(\rho^{0} \vec{E}+\vec{\rho} \times \vec{B}\right)-s \check{\gamma} \nabla G\right] d^{3} x,
\end{array}\right.
$$

where $\vec{E}$ and $\vec{B}$ include the intensity of external potential of $A^{\mu}$.

Substituting the classical approximation (2.9) into (3.22), we get the Newtonian second law for the spinor $\phi$

$$
\left\{\begin{array}{l}
\frac{d}{d t} p^{0}=e \vec{v} \cdot \vec{E}(t, \vec{X})+s W_{\gamma} \sqrt{1-v^{2}} \partial_{t} G(t, \vec{X})-\frac{d}{d t} E_{F} \\
\frac{d}{d t} \vec{p}=e(\vec{E}+\vec{v} \times \vec{B})-s W_{\gamma} \sqrt{1-v^{2}} \nabla G(t, \vec{X})
\end{array}\right.
$$

where $W_{\gamma}=\int_{R^{3}} \check{\gamma} d^{3} \bar{x}$. By the dynamical equation (3.3), we get[2]

$$
p^{\mu}=\int_{R^{3}} \Re\left\langle\phi^{+} \alpha^{\mu}\left(\hbar i \partial_{0}-e A_{0}\right) \phi\right\rangle d^{3} x .
$$

Assuming the spinor takes the energy eigenstate and moves smoothly, by (2.9) and (3.24) we find $p^{\mu} \propto u^{\mu}$, then by covariance we have

$$
p^{\mu}=m u^{\mu}
$$

where $m=\sqrt{p^{\mu} p_{\mu}}$ is the inertial mass of the spinor. (3.25) times (3.23), we have

$$
\begin{aligned}
\frac{1}{2} \frac{d}{d t}\left(p^{\mu} p_{\mu}\right) & =m s W_{\gamma} v^{\mu} \partial_{\mu} G-m W_{F} \frac{d}{d t} \ln \sqrt{1-v^{2}} \\
\frac{d}{d t} m & =\frac{d}{d t}\left[s W_{\gamma} G-W_{F} \ln \left(\sqrt{1-v^{2}}\right)\right]
\end{aligned}
$$

in which we used $\frac{d}{d t}=v^{\mu} \partial_{\mu}$. Integrating (3.27) we get the moving inertial mass $m$ of the spinor $\phi$

$$
m=m_{0}+s W_{\gamma} G(t, \vec{X})-W_{F} \ln \sqrt{1-v^{2}},
$$

where $m_{0}$ is constant static mass. Substituting (3.28) into (3.25) we get

$$
p^{\mu}=\left(m_{0}+s W_{\gamma} G-W_{F} \ln \sqrt{1-v^{2}}\right) u^{\mu} .
$$

Substituting (3.29), (3.16), (3.17) and (3.18) into (3.9), we finally get the complete massenergy relation for spinor $\phi$ as follows, which includes the contributions of all self-potentials 
of the spinor.

$$
\begin{aligned}
E & =E_{0}-\frac{M_{1} v^{2}}{\sqrt{1-v^{2}}}+\frac{W_{F}}{\sqrt{1-v^{2}}} \ln \frac{1}{\sqrt{1-v^{2}}}, \\
E_{0} & =\frac{M_{0}}{\sqrt{1-v^{2}}}, \\
M_{0} & =m_{0}+W_{F}+s W_{\gamma} G+W_{G}+W_{A}, \\
M_{1} & =W_{F}+\frac{1}{3}\left(W_{G}-W_{A}\right)+W_{b},
\end{aligned}
$$

where $M_{0}$ is the total static mass of $\phi$. By (3.30) and (3.31), we have

$$
\frac{E}{E_{0}}-1=\frac{W_{F}-2 M_{1}}{2 M_{0}} v^{2}+\frac{W_{F}}{4 M_{0}} v^{4}+O\left(v^{6}\right) .
$$

(3.34) can be tested by experiments, and 3 parameters $\left(M_{0}, M_{1}, W_{F}\right)$ can be determined.

\section{DISCUSSION AND CONCLUSION}

We derived the local Lorentz transformations for classical parameters and established the relationship between field theory and corresponding classical mechanics in a logical procedure. The energy-speed relations for each potential term are derived, and the classical mass of a particle is clearly defined. The subtle logic relationship between concepts of quantum mechanics and that of classical mechanics is overlooked previously and results in confusions and puzzles. The above results imply the following conclusions.

1. Classical mechanics once was a guidance to establish quantum mechanics. By analogy we define the concepts for quantum theory. However, from (3.1) to (3.23) and (3.29), we learn that, the Dirac equation (3.3) and field equations (3.4)-(3.6) are more profound and general theory than the classical one (3.23), because we can logically derive (3.23) from (3.1) under the assumption of mass-point model (2.9). Obviously, we cannot do the inverse operation. Analogy is not logic. When the more profound quantum theory goes to maturity, we should develop it relatively independent of classical mechanics. Otherwise, the overlapping concepts of two different systems certainly result in contradictions and confusions.

For spinor field $\phi$, we cannot specify its coordinate or momentum without a clear definition, because $x^{\mu}$ is just a presetting label system for space-time[14], rather than the coordinates of a particle. If we have not exact concepts for coordinate and momentum of the spinor, what is the meaning of the Heisenberg uncertainty relation? which is based on such concepts. Obviously this situation is caused by the residual classical 
picture of a particle in mind when we analyze the spinor field equation. As a matter of factor, except for the fields $\phi$ and $A^{\mu}$ and so on we have nothing else. $\phi$ and $A^{\mu}$ are basic properties for a matter system, and other concepts should be logically defined from them. In some cases a spinor acting like a particle is because interactions and environment make it so, and in this time we should clearly define classical concepts and then derive its simplified Newtonian mechanics as well as the conditions of validity as done above. Only in such procedure, confusions will be removed automatically and a lot of debates are superfluous. For (2.6), (2.7) and (3.29), how does one use the principle of uncertainty?

2. A more puzzling equation than (3.6) is the Schrödinger equation for $N$-electron,

$$
\hbar i \partial_{t} \Psi=\hat{\mathbf{H}} \Psi, \quad \hat{\mathbf{H}}=\sum_{k=1}^{N}\left(\frac{\hat{p}_{k}^{2}}{2 m}+e V\left(t, \vec{X}_{k}\right)+\frac{e^{2}}{8 \pi} \sum_{l \neq k} \frac{1}{\left|\vec{X}_{l}-\vec{X}_{k}\right|}\right) .
$$

In $\hat{\mathbf{H}}$ the coordinate $\vec{X}_{k}$ is quite similar to the classical one for mass-point, and we have $3 N$ coordinates for $N$ electrons. The detailed derivation and explanation for this equation and the origin of the $3 N$ coordinates is complex, which were given in $[2,15]$. We do not repeat it here. What we stress here is that, (4.1) is also the non-relativistic approximation of some juxtaposing Dirac equations. To explain the foundation of quantum mechanics, pure philosophical speculation is little helpful to solve the puzzles. Only if the logical structure between the concepts and equations is clarified, the puzzles automatically vanish.

3. Manifestly, field theories like (3.1) are compatible with general relativity. If we take the quantum theory to be essentially a field theory, and QED or QCD to be just an inconvenient and ambiguous computing procedure to solve field equation, then consistency problem vanishes immediately. Dirac equations have abundant solution spectra which could be solved only by reliable and systematic procedure constructed according to normal mathematics[13, 16, 17].

Furthermore, along this line of opinions, we naturally get a kind of unified field theory as follows[12]:

A1. The space-time is described by

$$
d \mathbf{x}=\widetilde{\gamma}_{\mu} d x^{\mu}=\gamma_{\alpha} \delta X^{\alpha}
$$

in which $\gamma_{\alpha}$ and $\widetilde{\gamma}_{\mu}$ satisfy the following $C \ell(1,3)$ Clifford algebra,

$$
\gamma_{\alpha} \gamma_{\beta}+\gamma_{\beta} \gamma_{\alpha}=2 \eta_{\alpha \beta}, \quad \widetilde{\gamma}_{\mu} \widetilde{\gamma}_{\nu}+\widetilde{\gamma}_{\nu} \widetilde{\gamma}_{\mu}=2 g_{\mu \nu}
$$


A2. The dynamics for a definite physical system is given by

$$
\partial \Psi=\mathcal{F}(\Psi), \quad \partial \equiv \widetilde{\gamma}^{\mu} \partial_{\mu}
$$

in which $\Psi=\left(\psi_{1}, \psi_{2}, \cdots, \psi_{n}\right)^{T}$, and $\mathcal{F}(\Psi)$ consists of some tensorial products of $\Psi$, so that the total equation is covariant.

A3. The solutions to (4.4) are singularity-free, that is, $\forall \psi_{k} \in L^{\infty}$.

The Dirac equation, Maxwell equation and Einstein equation all satisfy (4.4). By classifying $\Psi$ according to spin indices $s=\frac{1}{2}, 1,2$ we can derive these equations respectively as well as their coupling system. Under the constraint of A3, we have only limited choices for the interactive potentials. If we solve the dynamics (4.4) from the simplest system to more complicated ones, we will certainly reach the right results for all physical problems. In the standard model, the constraints of assumptions such as $S U(2)$ and $S U(3)$ are too strong and too narrow to contain enough physical objects, and the theory is trapped in complicated formalism. The more the constraints, the larger the risk and the less the truth.

4. In some textbooks, the relations (2.13)-(2.15) are directly derived via Lorentz transformation of the integrands and volume element relation $d^{3} x=\sqrt{1-v^{2}} d^{3} \bar{x}$. This calculation is only an approximation in general cases, because parameters in (2.15) depend on $\bar{t}$ in this case. It is accurate only for spinor at particle state. However, it is usually accurate enough for particles such as electron and proton due to the static mass energy much larger then energy exchanged with the environment.

Usually, the proper parameters have very simple form. For true vector, it usually takes the form $\bar{I}^{\mu}=\left(\bar{I}^{0}, 0,0,0\right)$, then we get

$$
I^{\mu}=\sqrt{1-v^{2}} \bar{I}^{0} u^{\mu}
$$

In some cases, the symmetrical true tensor $I^{\mu \nu}$ is given by

$$
I^{\mu \nu}=\sqrt{1-v^{2}}\left(\bar{K} u^{\mu} u^{\nu}+\bar{J} \eta^{\mu \nu}\right)
$$

where $\bar{K}, \bar{J}$ are constants.

5. By (3.29), (3.16), (3.17) and (3.18), we learn different potential has different energyspeed relation, so we can identify the interactive potentials by testing the fine structure of the energy-speed relation of a particle[18]. Especially, the existence of nonlinear potential $F$ can be determined, which is much important to disclose the nature of 
fundamental particles and dark matter. The linear Dirac equation is accurate for Hydrogen atom spectrum. How an electron balances its self-electromagnetic interaction can also be discovered by testing the mass-energy relation of an electron[18].

However, for normal particles such as electron, the numerical results[4]-[9] showed that the mass contributed by potentials $\left(W_{F}, W_{A}, W_{G}\right)$ and so on are much less than the static mass $m_{0} \approx \mu$, so Einstein's mass-energy relation holds to high precision if $v \ll c$.

6. By (3.28) or (3.29) we find the external scalar $G$ manifestly influence the inertial mass and momentum of a particle. This effect will seriously violate classical mechanics, so such a scalar field should be absent in Nature.

\section{Acknowledgments}

The author is grateful to his supervisor Prof. Ta-Tsien Li and Prof. Han-Ji Shang for their encouragement. It is my pleasure to thank the reviewer A for helping to clarify some subtle concepts and to correct a number of language errors.

[1] Einstein, A. Ann. der Phys. 18, 639-641 (1905).

[2] Y. Q. Gu, New Approach to N-body Relativistic Quantum Mechanics, Int. J. Mod. Phys. A22:2007-2020(2007), arXiv:hep-th/0610153

[3] Lee Smolin, The truble with physics, Spin Network, Ltd. 2006.

[4] R. Finkelsten, et al, Phys. Rev. 83(2), 326-332(1951)

[5] M. Soler, Phys. Rev. D1(10), 2766-2767(1970)

[6] M. Wakano, Prog. Theor. Phys. 35, 1117(1966)

[7] M. Soler, Phys. Rev. D8, 3424(1973)

[8] A. F. Randa, M. Soler, Phys. Rev. D8, 3430(1973)

[9] Y. Q. Gu, Some Properties of the Spinor Soliton, Adv in Appl. Clif. Alg. V8(1), 17-29(1998)

[10] Y. Q. Gu, A Cosmological Model with Dark Spinor Source, Int. J. Mod. Phys. A22:46674678(2007), gr-qc/0610147

[11] Y. Q. Gu, Some Subtle Concepts in Fundamental Physics, to appear in PHYSICS ESSAYS, arXiv:0901.0309

[12] Y. Q. Gu, The Quaternion Structure of Space-time and Arrow of Time, J. Mod. Phys. V3, $570-580(2012)$. 
[13] W. Greiner, B. Müller, Quantum Mechanics - Symmetries, Springer, 1994.

[14] Y. Q. Gu, Some Paradoxes in Special Relativity and resolutions, Adv in Appl. Clif. Alg. V21(1), 103-119(2011), arXiv:0902.2032

[15] Y. Q. Gu, The Electromagnetic Potential Among Nonrelativistic Electrons, Adv. Appl. Cliff. Alg. 9(1), 61-79(1999)

[16] Y. Q. Gu, A Procedure to Solve the Eigen Solution to Dirac Equation, Quant. Phys. Lett. 6, No. 3,161-163 (2017); arXiv:0708.2962

[17] Y. Q. Gu, Mass Spectrum of Dirac Equation with Local Parabolic Potential, arXiv:hep-th/0612214

[18] Y. Q. Gu, Test of Einstein's Mass-Energy Relation, arXiv:hep-th/0610189v3 